\title{
THE INTEGRAL OF AN INVARIANT UNIMODAL FUNCTION OVER AN INVARIANT CONVEX SET -AN INEQUALITY AND APPLICATIONS ${ }^{1}$
}

\author{
GOVIND S. MUDHOLKAR
}

1. Introduction and summary. It is well known that the integral $\int_{-a+\theta}^{a+\theta} f(x) d x, a>0$, of a nonnegative function $f(x)$ on the real line, which is unimodal, i.e., $f(k x) \geqq f(x), 0 \leqq k \leqq 1$, and symmetrical about the origin, is a monotonically decreasing function of $|\theta|$. An immediate probabilistic consequence of this is the fact that, if a random variable $X$ has a unimodal probability density function symmetric about the origin, and $Y$ is any independen tly distributed random variable, then $\operatorname{Pr}\{|X| \geqq a\} \leqq \operatorname{Pr}\{|X+Y| \geqq a\}$ for any real $a$. T. W. Anderson [1] has extended the aforementioned monotonicity property to integrals of functions on a Euclidean $n$-space $\mathfrak{L}_{n}$ by replacing the symmetric interval of the real line by a convex set of $\mathfrak{L}_{n}$ symmetric about the origin, and formulating the following definition of unimodality of functions on $\mathfrak{L}_{n}$.

Definition 1. A function $f(x)$ on $\mathscr{L}_{n}$ is said to be unimodal if the set $K_{u}=\{x \mid f(x) \geqq u\}$ is convex for each $u \geqq 0$.

More specifically, he has proved the following:

TheOREM 1. Let $E$ be a convex set in $\mathfrak{L}_{r}$, symmetric about the origin. Let $f(x)>0$ be a function such that (i) $f(x)=f(-x)$, (ii) $\{x \mid f(x)>u\}$ $=K_{u}$ is convex for every $u,(0<u<\infty)$, and (iii) $\int_{E} f(x) d x<\infty$ (in the Lebesque sense). Then

$$
\int_{E} f(x+k y) d x \geqq \int_{E} f(x+y) d x
$$

for $0 \leqq k \leqq 1$.

Anderson has also discussed some analogues of the probability inequality mentioned above and many other probabilistic and statistical applications.

In $\$ 2$ we have obtained a generalization of the Theorem 1 by relaxing the condition of symmetry about the origin, on the function $f$ and the set $E$, to a restriction of invariance with respect to finite groups of linear transformations of $\mathfrak{L}_{n}$, and we have indicated the analogues of some of the probability inequalities in [1].

Received by the editors March 21, 1966.

${ }^{1}$ Research supported in part by NSF Grant GP 5801. 
In $\$ 3$ we have discussed some particular cases by considering invariance with respect to the group of reflections in the origin, the permutation group, and the cyclic permutation group in $n$-space. It has been shown that the integral of a symmetric unimodal function over a symmetric convex region, is an $S$-concave (in Ostrowski's sense) function of the translation parameter.

In the final $\S 4$, we have stated and have outlined a somewhat different proof of a slightly different version of the inequality without assuming the group of transformations to be finite.

2. The inequality with invariance w.r.t. a finite group $G$. Let $G=\left\{g_{i}\right.$, $i=1,2, \cdots, N\}$ be a finite group of Lebesgue measure-preserving linear transformations of $\mathfrak{L}_{n}$ onto $\mathfrak{L}_{n}$. Let $E$ be a convex set of $n$ space, invariant under $G$, or $G$-invariant, i.e. $x \in E$ implies $g_{i} x \in E$, $i=1,2, \cdots, N$. Let $f(x) \geqq 0$ be a function on $n$-space satisfying

(i) the unimodality condition: $\{x \mid f(x) \geqq u\}=K_{u}$ is convex for every $u, 0<u<\infty$,

(2) (ii) $G$-invariance condition: $f\left(g_{i} x\right)=f(x), \quad i=1,2, \cdots, N$, for each $x$ in $\mathfrak{L}_{n}$, and

(iii) $\int_{E} f(x) d x<\infty$ in the Lebesgue sense.

For a set $\alpha=\left\{\alpha_{1}, \alpha_{2}, \cdots, \alpha_{N}\right\}, \alpha_{i} \geqq 0, i=1,2, \cdots, N, \sum_{i=1}^{N} \alpha_{i}=1$, and a vector $y$ of $n$-space let us define

$$
\alpha(y)=\sum_{i=1}^{N} \alpha_{i} g_{i} y .
$$

Then we have, as a generalization of the Theorem 1, the following:

THEOREM 2. For each set $\alpha=\left\{\alpha_{1}, \cdots, \alpha_{N}\right\}, \alpha_{i} \geqq 0, \sum \alpha_{i}=1$, and vector $y$ of $\mathfrak{L}_{n}$ we have

$$
\int_{E} f(x+\alpha(y)) d x \geqq \int_{E} f(x+y) d x,
$$

where $f \geqq 0$ and $E$ are $G$-invariant, $f$ is unimodal, $E$ is convex and $\alpha(y)$ is defined by (3), provided $\int_{E} f(x) d x<\infty$.

Proof. We have to show, equivalently, that

$$
\int_{E+\alpha(y)} f(x) d x \geqq \int_{E+\boldsymbol{y}} f(x) d x,
$$

where $E+y$ is the set $E$ translated by the vector $y$. Now it is easy to verify that, because of the convexity of $E$ and $K_{u}$ 


$$
\{E+\alpha(y)\} \cap K_{u} \supset \sum_{i=1}^{N} \alpha_{i}\left[\left\{E+g_{i} y\right\} \cap K_{u}\right],
$$

where the summation symbol $\sum$ on the right hand side of the inclusion relation corresponds to the sum, called the Minkowski sum [4], defined by

$$
A+B=\{a+b \mid a \in A, b \in B\}
$$

for any two sets $A, B$ of $\mathfrak{L}_{n}$, and where multiple $c A$ of a set $A$ of $\mathfrak{L}_{n}$ by a real $c$ is defined by $c A=\{c a \mid a \in A\}$. Let $\mu(\cdot)$ be the Lebesgue measure of sets in $\mathfrak{L}_{n}$. Then we have by the Brunn-Minkowski theorem $[2],[3],[4]$,

$$
\mu^{1 / n}\left(\sum_{i=1}^{N} \alpha_{i}\left[\left\{E+g_{i} y\right\} \cap K_{u}\right]\right) \geqq \sum_{i=1}^{N} \alpha_{i} \mu^{1 / n}\left(\left\{E+g_{i} y\right\} \cap K_{u}\right) .
$$

But because of the invariance of $f$ and $E$ and linearity and measure preserving properties of the transformations $g_{i}, i=1,2, \cdots, N$, we have

$$
\mu\left(\left\{E+g_{i} y\right\} \cap K_{u}\right)=\mu\left(\{E+y\} \cap K_{u}\right) .
$$

Combining (6), (8) and (9) we get

$$
H(u)=\mu\left(\{E+\alpha(y)\} \cap K_{u}\right) \geqq \mu\left(\{E+y\} \cap K_{u}\right)=H^{*}(u) .
$$

Because of the definition of Lebesgue-Stieltjes integrals we can write

$$
\int_{E+\alpha(y)} f(x) d x-\int_{E+y} f(x) d x=\int_{0}^{\infty} u d\left[H^{*}(u)-H(u)\right] .
$$

The right hand side of (11) is nonnegative, which may be verified by using integration by parts as in the proof of the Theorem 1 of [1]. This completes the proof of the Theorem 2 .

As in [1], it may be noted that we obtain strict inequality in (4) if, and only if, for at least one $u, H(u)>H^{*}(u)$, since $H(u)$ is continuous on the left. For $H(u)=H^{*}(u)$ we need equality in (8), which is a consequence of the Brünn-Minkowski theorem. The condition for equality may, therefore, be stated as:

Corollary 1. In the Theorem 2, the equality in (4) holds if, and only if, $\left(E+g_{i} y\right) \cap K_{u}$ are similarly oriented for each $u$.

CoROllary 2. If the probability density function $f(x)$ of a random $n$-vector $X$ satisfies the conditions (2) and $E$ is a convex set of $n$-space invariant under $G$, then for any n-vector $y$ and set $\alpha, \operatorname{Pr}\{X+\alpha(y) \in E\}$ 
$\geqq \operatorname{Pr}\{X+y \in E\}$. Furthermore, if $h(x)$ is a $G$-invariant function such that $\{x \mid h(x) \leqq v\}$ is convex, then $\operatorname{Pr}\{h(X+\alpha(y)) \leqq v\} \geqq \operatorname{Pr}\{h(X+y)$ $\leqq v\}$.

The proof of the following corollary is analogous to the proof of the Theorem 2 of [1].

CoROllary 3. Let the probability density function $f(x)$ of a random $n$-vector $X$ satisfy the conditions (2) and let $Y$ be any independently distributed random $n$-vector. Then for any set $\alpha=\left\{\alpha_{1}, \cdots, \alpha_{N}\right\}$, $\alpha_{1} \geqq 0, \sum \alpha_{i}=1$, and any convex $G$-invariant set $E$ of $n$-space

$$
\operatorname{Pr}\{X+\alpha(Y) \in E\} \geqq \operatorname{Pr}\{X+Y \in E\} .
$$

Furthermore, if $h(x)$ is a G-invariant function such that $\{x \mid h(x) \leqq v\}$ is convex, then

$$
\operatorname{Pr}\{h(X+\alpha(Y)) \leqq v\} \geqq \operatorname{Pr}\{h(X+Y) \leqq v\} .
$$

3. Some particular cases. (i) If the group $G$ in $\$ 2$ is the group of reflections in the origin the Theorem 2 reduces to the above stated Theorem 1 of Anderson [1].

(ii) An important particular case of the Theorem 2 is obtained if the group $G$ is the permutation group in $\mathscr{L}$.

Definition 2 (Ostrowski [6]). A function $G(y)$ on $\mathfrak{L}_{n}$ is said to be $S$-concave if, for each doubly stochastic matrix $S$, of order $n$, and each $y$ in $\mathfrak{L}_{n}$

$$
G(S y) \geqq G(y) .
$$

Now Birkhoff's theorem [5] states that the set of doubly stochastic matrices of order $n$ is a convex polyhedron with $N=n$ ! permutation matrices $P_{i}, i=1,2, \cdots, N$, as the vertices. Thus every doubly stochastic matrix $S=\sum_{i=1}^{N} \alpha_{i} P_{i}$ for some set $\alpha=\left\{\alpha_{1}, \cdots, \alpha_{N}\right\}$, $\alpha_{i} \geqq 0, \sum_{i=1}^{n} \alpha_{1}=1$. Hence we have the following Theorem 3 as a particular case of the Theorem 2 .

THEOREM 3. Let a function $f(x) \geqq 0$ on $\mathfrak{L}_{n}$ be symmetric (w.r.t. permutations) and satisfy the conditions (i) and (iii) of (2). Let $E$ be a convex, symmetric (w.r.t. permutations) set of $\mathfrak{L}_{n}$. Then $\int_{E} f(x+y) d x$ is an S-concave function of $y$, i.e.,

$$
\int_{E} f(x+S y) d x \geqq \int_{E} f(x+y) d x,
$$

for any doubly stochastic matrix $S$. 
It is also well known [6] that, given two $n$-vectors $y$ and $z$, there exists a doubly stochastic matrix $S$, such that $z=S y$ if, and only if,

$$
\begin{aligned}
& z_{(1)}+\cdots+z_{(k)} \leqq y_{(1)}+\cdots+y_{(k)}, \quad k=1,2, \cdots, n-1, \\
& z_{(1)}+\cdots+z_{(n)}=y_{(1)}+\cdots+y_{(n)},
\end{aligned}
$$

where $y_{(i)}$ and $z_{(i)}, i=1,2, \cdots, n$ are the coordinates of $y$ and $z$, ordered in nonincreasing order of magnitude. The conclusion (15) of the Theorem 3 may thus be expressed as: For any two vectors $y$ and $z$ of $n$-space, satisfying (16) we have

$$
\int_{E} f(x+z) d x \geqq \int_{E} f(x+y) d x .
$$

CoROllaRy 4. If the probability density function $f(x)$ of a random $n$-vector $X$ is unimodal and symmetric w.r.t. permutations of the coordinates of $x$, and $Y$ is an independently distributed random vector then for any convex symmetric set $E$ of $\mathfrak{L}_{n}$, and any doubly-stochastic matrix $S$, we have

$$
\operatorname{Pr}\{X+S Y \in E\} \geqq \operatorname{Pr}\{X+Y \in E\} .
$$

Furthermore, if $h$ is a symmetric function such that $\{x \mid h(x) \leqq v\}$ is convex then

$$
\operatorname{Pr}\{h(X+S Y) \leqq v\} \geqq \operatorname{Pr}\{h(X+Y) \leqq v\} .
$$

(iii) Now let the transformation group of $\$ 2$ be the group of the cyclic permutations of $n$ coordinates. One has the matrix representation for this group as $g_{i}=p^{i-1}, i=1,2, \cdots, n$, where $P$ is a permutation matrix given by

$$
P=\left[\begin{array}{lllll}
0 & 0 & \cdots & 0 & 1 \\
1 & 0 & \cdots & 0 & 0 \\
0 & 1 & \cdots & 0 & 0 \\
\cdot & \cdot & \cdot & \cdot & . \\
0 & 0 & \cdots & 1 & 0
\end{array}\right]
$$

Thus for any vector $y$ of $\mathfrak{L}_{n}$ and a set $\alpha=\left\{\alpha_{1}, \alpha_{2}, \cdots, \alpha_{n}\right\}, \alpha_{i} \geqq 0$, $i=1,2, \cdots, n, \sum \alpha_{i}=1$ we have

$$
\alpha(y)=\sum_{i=1}^{n} \alpha_{i} g_{i} y=\sum_{i=1}^{n} \alpha_{i} P^{i+1} y=C(\alpha) y,
$$

where $C(\alpha)$ is the doubly stochastic circulant matrix given by $C(\alpha)$ $=\left(c_{i j}\right), c_{i j}=\alpha_{k}, k=k(i, j)=i+j-1 \bmod (n)$ or more explicitly, 


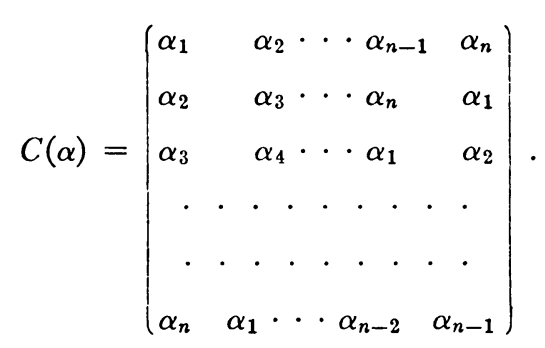

Also, it is easy to verify that the set of all doubly stochastic circulant matrices form a convex polyhedron with the permutation matrices $p^{i-1}, i=1,2, \cdots, n$, as the vertices. We have, therefore, the following Theorem 4 as another particular case of the Theorem 2.

TheOREM 4. Let a function $f(x) \geqq 0$ on $n$-space by unimodal and invariant under cyclic permutations. Let $E$ be a convex set of $n$-space symmetric with respect to cyclic permutations. Let $\int_{E} f(x) d x<\infty$. Then for any doubly stochastic circulant matrix $C$ and any vector $y$ of $n$-space we have

$$
\int_{E} f(x+C y) d x \geqq \int_{E} f(x+y) d x .
$$

It is easy to write down analogues of the Corollary 4 for this case. One may similarly write down, with ease, the particular case of the Theorem 2 when the function $f(x)$ and the set $E$ are invariant under the group of $2^{n}$ reflections in the coordinate planes.

4. The inequality. We shall now outline a somewhat different proof of a somewhat different and generalized version of the inequality of $\$ 2$ without the finiteness condition on the group $G$.

Theorem 5. Let $G=\{g\}$ be a group of linear Lebesgue measurepreserving transformations of $\mathfrak{L}_{n}$ onto $\mathfrak{L}_{n}$. Let $E$ be a convex, $G$-invariant region of $\mathfrak{L}_{n}$. Let $f$ be a nonnegative real-valued, $G$-invariant and unimodal function on $\mathfrak{L}_{n}$. Then for arbitrary $y$ in $\mathfrak{L}_{n}$ we have

$$
\int_{E} f(x+z) d x \geqq \int_{E} f(x+y) d x,
$$

where $z$ is any point in the convex-hull of the G-orbit of $y$.

Proof. The theorem can be proved along the lines of the proof of the Theorem 2, by using the generalized version of the BrünMinkowski theorem due to Dinghas [2], [3], [4]. However, the argument may be simplified as follows, by using a twist suggested by 
Kemperman in a personal communication.

The crucial step in the proof of the Theorem 2 is the statement (10), which holds without requiring $G$ to be finite. To see this let us fix $y$ and write,

$$
Z=\{z \mid \phi(z) \geqq \phi(y)\}
$$

where

$$
\phi(z)=\mu^{1 / n}\left((E+z) \cap K_{u}\right) .
$$

Then by the Brünn-Minkowski theorem it follows that,

$$
\phi\left(\lambda z_{1}+(1-\lambda) z_{2}\right) \geqq \lambda \phi\left(z_{1}\right)+(1-\lambda) \phi\left(z_{2}\right), \quad \text { for } 0 \leqq \lambda_{1} \leqq 1 .
$$

Hence for any $y$ in $\mathfrak{L}_{n}$ the set $Z$ of (20) is convex. Furthermore as in the proof of the Theorem 2 it can be verified that $g y \in Z$ for each $g \in G$. Therefore, for any point $z$ in the convex-hull of $\{g y \mid g \in G\}$ we have

$$
\mu\left((E+z) \cap K_{u}\right) \geqq \mu\left((E+y) \cap K_{u}\right),
$$

which is analogous to the statement (10). The proof from here on is the same as the proof of the Theorem 2.

The analogue of the Corollary 2 for the Theorem 5 is easy to formulate. A group $G$ of special interest in probability and statistics is the group of orthogonal transformations. The $G$-orbit of any $y$ for this group is the sphere $\sum_{i=1}^{n} x_{i}^{2}=\|y\|^{2}$ in $\mathfrak{L}_{n}$. This special case may be studied as in $\$ 3$ without any difficulty.

Acknowledgment. I wish to record thanks to J. H. B. Kemperman for some valuable discussions and suggestions.

\section{REFERENCES}

1. T. W. Anderson, The integral of a symmetric unimodal function over a symmetric convex set and some probability inequalities, Proc. Amer. Math. Soc. 6 (1955), 170-176.

2. A. Dinghas, Sur une généralisation du théorème de Lusternik concernant des familles continues des ensembles, C. R. Acad. Sci. Paris 239 (1954), 575-576.

3. - Demonstration du theorème de Brünn-Minkowski pour des familles continues d'ensembles, C. R. Acad. Sci. Paris 239 (1954), 605-607.

4. - Minkowskische Summen und integrale superadditive Mengenfunctionale. Isoperimetrische Ungliechungen, Memorial des Sciences Mathematiques CXLIX, Gauthier-Villars, Paris, 1961.

5. M. Marcus and H. Minc, A Survey of matrix theory and matrix inequalities, Allyn and Bacon, Boston, 1964.

6. A. Ostrowski, Sur quelques applications des fonctions convexes et concaves au sens de I. Schur, J. Math. Pures Appl. (9) 31 (1952), 253-292.

THE UNIVERSITY OF Rochester 